\title{
Phase breaking in three-terminal contacted single-walled carbon nanotube bundles
}

\author{
V. Krstić* S. Roth, and M. Burghard \\ Max-Planck-Institut für Festkörperforschung, Heisenbergstrasse 1, D-70659 Stuttgart, Germany
}

(Received 10 August 2000)

\begin{abstract}
The three-terminal electrical transport through single-walled carbon nanotube bundles with low resistive metal contacts is investigated at room temperature. After correcting for the lead resistance, two-probe resistances close to the value expected for a metallic single-walled carbon nanotube are found. Analysis of the experimental data in the frame of the Landauer-Büttiker formalism reveals the phase- and momentumrandomizing effect of the third electrode, which is at floating potential, on the quasiballistic transport. Within this model, the phase-coherence length of the charge carriers is estimated to be $\approx 300 \mathrm{~nm}$ at room temperature.
\end{abstract}

The potential application of carbon nanotubes (CNT's) as molecular wires in submicron devices has initiated a variety of conductance experiments. Phenomena like Coulomb blockade $^{1,2}$ and signatures of Luttinger liquids ${ }^{3}$ in singlewalled carbon nanotubes (SWCNT's), as well as AharanovBohm oscillations, ${ }^{4}$ quantized conductance and quasiballistic transport at room temperature (RT) in multiwalled carbon nanotubes (MWCNT's) were reported. ${ }^{5}$ Except for the observations of Coulomb blockade, a low contact resistance between leads and the CNT is required in such experiments.

In the present study, electron-beam lithography (EBL) was used to contact SWCNT's with electrodes on top, similar to Ref. 3. Electrode arrays consisting of three equidistant stripes were prepared from AuPd (40 wt \%/60 wt \%). The electrical transport investigations at room temperature presented here focus on samples with SWCNT bundles, which are connected by three low-Ohmic contacts, in contrast to recent measurements with only two contacts. 3,6

For sample preparation, ${ }^{7}$ arc-discharge SWCNT raw material was dispersed by ultrasonic treatment in aqueous surfactant solution, and purified by centrifugation. As substrate, an As doped $\mathrm{Si}$ wafer with a thermally grown $\mathrm{SiO}_{2}$ layer was used. Before adsorption of the SWCNT's, the substrate was treated with a 0.1 wt $\%$ aqueous solution of 3-(aminopropyl)triethoxysilane for $2 \mathrm{~min}$. In order to perform EBL (EBL 100 system, LEICA) on top of the SWCNT's, a two-layer resist system was used. ${ }^{2}$ After EBL, AuPd (thickness $\approx 17 \mathrm{~nm}$ ) or Au (thickness $\approx 24 \mathrm{~nm}$ ) was thermally evaporated on the substrate at a base pressure of $p \approx 10^{-7}$ mbar using a rate of $1 \AA$ / .

Figure 1 shows a scanning force microscope (SFM) image (Tapping Mode, Digital Instruments, Nanoscope IIIa) of a typical sample we have investigated. A thin SWCNT bundle is connected to three AuPd electrodes. The electrode stripes are separated by $100 \mathrm{~nm}$, and are approximately $100 \mathrm{~nm}$ in width. The electrical transport measurements were performed in vacuum ( $p \leqslant 10^{-3}$ mbar) at RT.

In the upper inset of Fig. 1, the SFM height profile across electrode stripe 2 is shown. The contour of the bundle can be clearly detected in the profile, revealing a height that coincides with the height of the uncovered parts of the bundle $(\approx 3 \mathrm{~nm})$. This result indicates that its structural integrity is preserved during metal evaporation due to the high mechani- cal stability of the SWCNT's. The difference in work functions of the SWCNT and the AuPd may shift locally the Fermi energy of the SWCNT, but energy gaps in the local density of states due to tube bending, ${ }^{8}$ which would disturb the charge carrier transport, are unlikely in our configuration.

The current-voltage characteristics of the SWCNT bundle are presented in Fig. 2. The measured resistances obey the following relations: $R_{I}=R_{I I},\left\{R_{I}, R_{I I}\right\}<R_{I I I}$ and $R_{I}+R_{I I}$ $>R_{I I I}$. Between two neighboring electrodes, the twoterminal resistances $R_{i}=10.5 \mathrm{k} \Omega \pm 0.1 \mathrm{k} \Omega(i=I, I I)$ at $\mathrm{RT}$ are slightly larger than the values reported by other groups [around $8.6 \mathrm{k} \Omega,{ }^{3,6}$ which was identified as $(1 / 3) h / e^{2}$ ]. To account for the lead geometry and to further analyze the data applying an appropriate model, we determined the resistance $R_{\Lambda}$ of the two-terminal electrode 2 to $R_{\Lambda}=4.1 \mathrm{k} \Omega \pm 0.1 \mathrm{k} \Omega$.

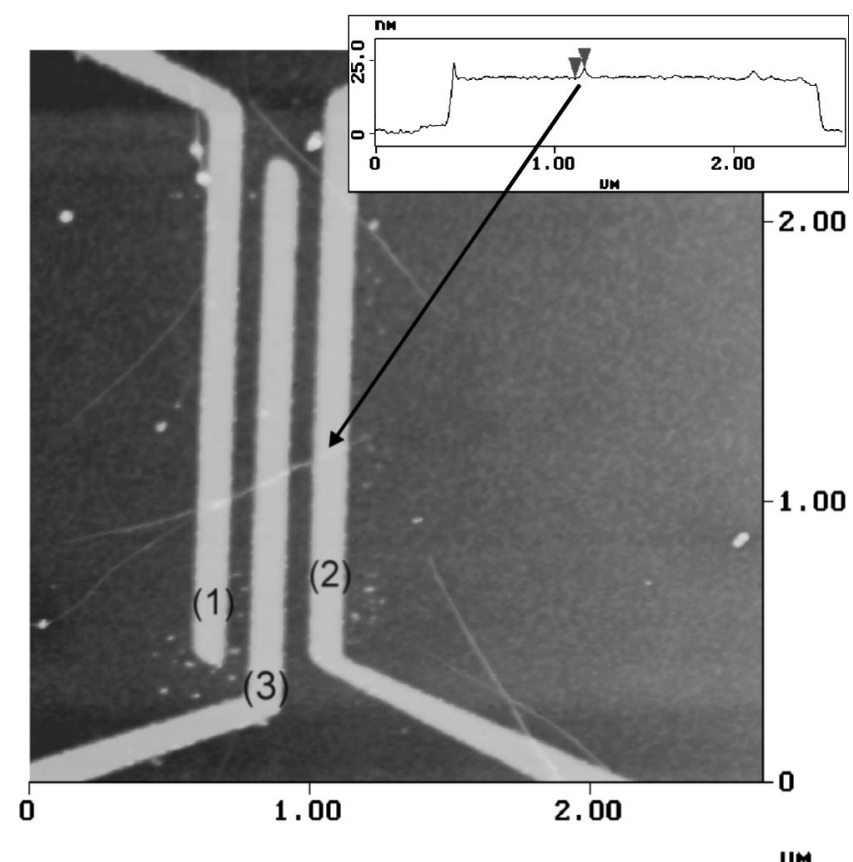

FIG. 1. SFM image of three AuPd electrode lines contacting a thin SWCNT bundle (height $\approx 3 \mathrm{~nm}$ ). Upper right inset: crosssectional analysis along electrode stripe 2 . The profile of the bundle clearly presses through the electrodes, revealing a height of about 3 nm (see triangles). 


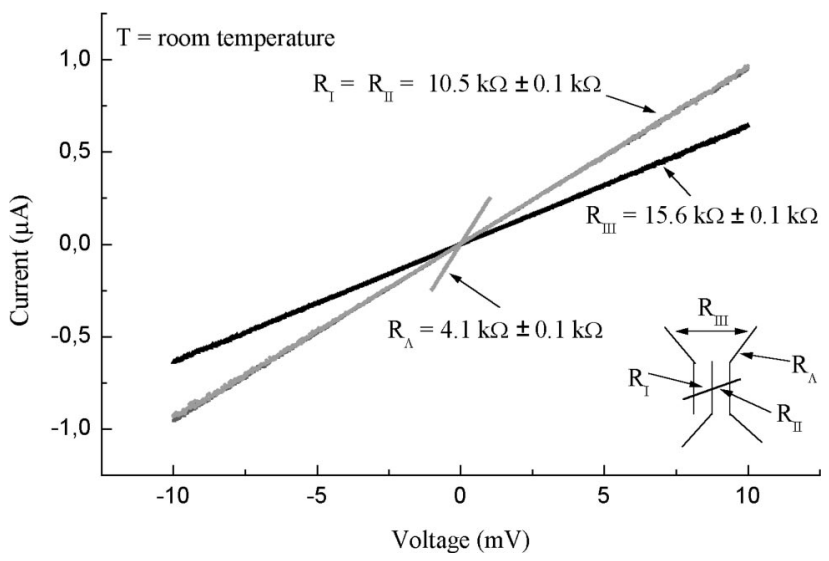

FIG. 2. Current-voltage characteristics at room temperature. The resistances $R_{j}(j=I, I I, I I I)$ are assigned to the respective electrode pairs. Bottom right: schematic drawing of the electrode array.

This value should be subtracted from the total measured resistance. Since the electrode structure is symmetric, the measured resistances $R_{i}(i=I, I I, I I I)$ are thus changed to $R_{I}^{(c)}$ $=R_{I I}^{(c)}=6.4 \mathrm{k} \Omega \pm 0.2 \mathrm{k} \Omega$ and $R_{I I I}^{(c)}=11.6 \mathrm{k} \Omega \pm 0.3 \mathrm{k} \Omega$, which we attribute to the two-terminal resistance of the SWCNT bundle. In view of the identity $R_{I}^{(c)}=R_{I I}^{(c)}$ and $R_{I}^{(c)}+R_{I I}^{(c)}$ $>R_{I I I}^{(c)}$ we assume that charge transport occurs through a single SWCNT in the bundle. ${ }^{9}$ For comparison, the twoterminal resistance $R_{\text {theo }}$ of a metallic SWCNT at RT can be described by the Landauer-Büttiker formalism in general as $^{10-13}$

$$
R_{\text {theo }}=\left(V_{1}-V_{2}\right)\left\{\frac{2 e}{h} \sum_{i} T_{i}\left(\mu_{1}-\mu_{2}\right)\right\}^{-1} \equiv \frac{h}{2 e^{2}} T_{\text {eff }}^{-1},
$$

assuming charge transport to be quasiballistic, phase coherent, ${ }^{14,15}$ and neglecting the thermal broadening due to the quasi-one-dimensional density of states of CNT's. ${ }^{12,13,16,17} V_{j}$ is the potential and $\mu_{j}=e V_{j}(j=1,2)$ is the electrochemical potential of electrode $j, \Sigma_{i}$ is the sum over all conducting channels $i$ and $T_{i}$ its transmission probability, respectively. ${ }^{12,13}$ In the case of metallic SWCNT's and strong electronic coupling to the terminals, ${ }^{18}$ one has two spin-polarized conducting channels contributing to the conductance of the system with $T_{i}=1$ for $i=1,2 .^{14,15}$ Therefore, $T_{\text {eff }}$ equals 2 and thus $R_{\text {theo }}=(1 / 4) h / e^{2} \approx 6.5 \mathrm{k} \Omega$, which is in very good agreement with the values of $R_{I}^{(c)}$ and $R_{I I}^{(c)}$ observed in our experiment. This agreement is a strong indication of quasiballistic, phase-coherent transport at RT as it was already proposed for SWCNT's on the basis of earlier experiments. ${ }^{9}$ Hence, the phase coherence length $l_{\varphi}$ is found to be at least $100 \mathrm{~nm}$, which is the distance of two neighboring electrodes.

The relations $\left\{R_{I}^{(c)}, R_{I I}^{(c)}\right\}<R_{I I I}^{(c)}$, and in particular $R_{I}^{(c)}$ $+R_{I I}^{(c)}>R_{I I I}^{(c)}$, reveal that the usual series resistor model does not apply, for which $R_{I}^{(c)}+R_{I I}^{(c)} \leqslant R_{I I I}^{(c)}$ would be expected. Following the Landauer-Büttiker formalism, electrode 3 represents a probe at the floating potential which acts as an inelastic scatter randomizing the momentum and phase of the charge carriers. ${ }^{19,20}$ As a consequence, the current between

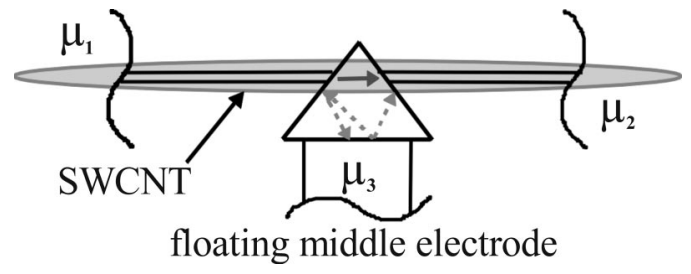

FIG. 3. Schematic illustration of the action of electrode 3 at floating potential. The black lines within the SWCNT (gray) represent the two conducting channels. The scattering process is indicated as a triangle. Coherently passing charge carriers are denoted by the continuous and scattered ones by the broken gray arrows. $\mu_{j}$ $(j=1,2,3)$ are the electrochemical potentials of the electrodes and the voltage applied is $e \mu_{1}-e \mu_{2}$.

electrode 1 and electrode 2 consists of a phase-coherent and an incoherent part. ${ }^{12,20}$ The situation is schematically depicted in Fig. 3.

Charge carriers flowing directly from electrode 1 to electrode 2 represent the coherent part, whereas the remaining charge carriers are phase randomized upon scattering into electrode 3, and then are emitted back in the SWCNT. In addition, the carriers are not necessarily emitted back into the same conduction channel from which they originated. In effect, electrode 3 impedes the propagation of charge carriers $^{20}$ along the SWCNT. The current through such a system can be expressed as ${ }^{20}$

$$
I=\frac{2 e}{h}\left\{\sum_{\alpha} T_{\alpha}+\frac{\sigma_{i} \cdot \sigma_{a}}{\sigma_{i}+\sigma_{a}}\right\}\left(\mu_{1}-\mu_{2}\right) \equiv \frac{2 e^{2}}{h} T_{\mathrm{eff}}\left(V_{1}-V_{2}\right),
$$

where $\Sigma_{\alpha} T_{\alpha}$ is the sum over all transmission probabilities $T_{\alpha}$ ( $\alpha$ are the coherent channels) through the scatterer. The quantity $\sigma_{i}\left(\sigma_{a}\right)$ sums over all transmission probabilities from electrode 3 into the SWCNT in (against) the overall direction of the current flow. From the measured current at $\left|V_{1}-V_{2}\right|=10 \mathrm{mV}$, one finds $T_{\text {eff }} \approx 1.2$, which is significantly smaller than in the two-terminal case. The difference in the work function of the metal and the SWCNT is unlikely to form a reflective potential barrier for the conducting channels. Otherwise higher values than $6.4 \mathrm{k} \Omega \pm 0.2 \mathrm{k} \Omega$ for $R_{I}^{(c)}$ and $R_{I I}^{(c)}$ should be observed for a metallic SWCNT. On that basis, $l_{\varphi}$ can be estimated from the distance between electrode 1 and electrode 2 to be about $300 \mathrm{~nm}$ at RT, which is of the same order as reported at low temperatures for MWCNT's $(\approx 3 \mathrm{~K})$ and SWCNT rings $(\approx 6 \mathrm{~K}) .^{21,22}$

Similar behavior has been observed for other samples, including SWCNT's connected to three Au electrodes. Interestingly, the ratios $R_{i} / R_{I I I}(i=I, I I)$ were of the same magnitude $(\approx 0.6)$ as in the case of AuPd electrodes.

In conclusion, the SFM investigations demonstrate the high mechanical stability of SWCNT's against metal evaporation on top. The observed electrical transport behavior provides evidence that SWCNT's, similar to MWNT's, ${ }^{5}$ can behave as phase-coherent, quasiballistic conductors at RT for small applied voltages. An additional floating electrode with strong electronic coupling acts as phase-randomizing, inelastic scatterer that impedes the phase-coherent transport, which is reflected in the lower effective transmission probability of 
about 1.2 compared to the value of 2 in the undisturbed case. A room-temperature phase-coherence length of about 300 $\mathrm{nm}$ can be estimated from those results. These findings demonstrate that in the future development of CNT-based de- vices, the influence of all connected electrodes should be taken into account. In addition, different electrode materials could provide specific tuning possibilities, depending on the nature of their electronic coupling to the CNT's.
*Corresponding author. Email address: krstic@klizix.mpistuttgart.mpg.de

${ }^{1}$ M. Bockrath, D. H. Cobden, P. L. McEuen, N. G. Chopra, A. Zettel, A. Thess, and R. E. Smalley, Science 275, 1922 (1997).

${ }^{2}$ V. Krstić, J. Muster, G. S. Duesberg, G. Philipp, M. Burghard, and S. Roth, Synth. Met. 110, 245 (2000).

${ }^{3}$ M. Bockrath, D. H. Cobden, J. Lu, A. G. Rinzler, R. E. Smalley, L. Balents, and P. L. McEuen, Nature (London) 397, 598 (1999).

${ }^{4}$ A. Bachthold, C. Strunk, J.-P. Salvetat, J.-M. Bonard, L. Forro, T. Nussbaumer, and C. Schönenberger, Nature (London) 397, 673 (1999)

${ }^{5}$ S. Frank, P. Poncharal, Z. L. Wang, and W. A. de Heer, Science 280, 1744 (1998).

${ }^{6}$ J. Nygard, D. H. Cobden, M. Bockrath, P. L. McEuen, and P. E. Lindelof, Appl. Phys. A: Mater. Sci. Process. 69, 297 (1999).

${ }^{7}$ V. Krstić, G. S. Duesberg, J. Muster, M. Burghard, and S. Roth, Chem. Mater. 10, 2338 (1998).

${ }^{8}$ A. Rochefort, P. Avouris, F. Lesage, and D. R. Salahub, Phys. Rev. B 60, 13824 (1999).

${ }^{9}$ A. Bezryadin, A. R. M. Verschueren, S. J. Tans, and C. Dekker, Phys. Rev. Lett. 80, 4036 (1998).

${ }^{10}$ R. Landauer, Philos. Mag. 21, 863 (1970).

${ }^{11}$ M. Büttiker, Phys. Rev. B 32, 1846 (1985).
${ }^{12} \mathrm{~S}$. Datta, Electronic Transport in Mesoscopic Systems (Cambridge University Press, Cambridge, 1995).

${ }^{13}$ Y. Imry, Introduction to Mesoscopic Physics (Oxford University Press, Oxford, 1997).

${ }^{14}$ C. T. White and T. N. Todorov, Nature (London) 393, 240 (1998).

${ }^{15}$ M. P. Anantram and T. R. Govindan, Phys. Rev. B 58, 4882 (1998).

${ }^{16}$ T. W. Odom, J.-L. Huang, P. Kim, and C. M. Lieber, J. Phys. Chem. B 2000, 2794 (2000).

${ }^{17}$ Additionally, the maximum applied voltage of $+10 \mathrm{mV}$ ensures it to be in the linear-response regime.

${ }^{18}$ M. P. Anantram, S. Datta, and Y. Xue, Phys. Rev. B 61, 14219 (2000).

${ }^{19}$ M. Büttiker, Phys. Rev. Lett. 57, 1761 (1986).

${ }^{20}$ M. Büttiker, Phys. Rev. B 33, 3020 (1986).

${ }^{21}$ A. Bachthold, C. Strunk, C. Schönenberger, J.-P. Salvetat, and L. Forro, in Electronic Properties of Novel Materials: Progress in Molecular Nanostructures, XII International Winterschool, Kirchberg 1998, edited by H. Kuzmany, J. Fink, M. Mehring, and S. Roth, AIP Conference Proceedings No. 442 (Woodbury, New York, 1998), pp. 65-68.

${ }^{22}$ H. R. Shea, R. Martel, and Ph. Avouris, Phys. Rev. Lett. 84, 4441 (2000). 